\title{
e-ASPECTS Correlates with and Is Predictive of Outcome after Mechanical Thrombectomy
}

\author{
(D). Pfaff, (D) C. Herweh, (DS. Schieber, (D) S. Schönenberger, (D). Bösel, (DP.A. Ringleb, (D) M. Möhlenbruch,
} (D) M. Bendszus, and (DS. Nagel Oㄹ

\begin{abstract}
BACKGROUND AND PURPOSE: The e-ASPECTS software is a tool for the automated use of ASPECTS. Our aim was to analyze whether baseline e-ASPECT scores correlate with outcome after mechanical thrombectomy.

MATERIALS AND METHODS: Patients with ischemic strokes in the anterior circulation who were admitted between 2010 and 2015 , diagnosed by CT, and received mechanical thrombectomy were included. The ASPECTS on baseline CT was scored by e-ASPECTS and 3 expert raters, and interclass correlation coefficients were calculated. The e-ASPECTS was correlated with functional outcome (modified Rankin Scale) at 3 months by using the Spearman rank correlation coefficient. Unfavorable outcome was defined as mRS 4-6 at 3 months, and a poor scan was defined as e-ASPECTS $0-5$.
\end{abstract}

RESULTS: Two hundred twenty patients were included, and $147(67 \%)$ were treated with bridging protocols. The median e-ASPECTS was 9 (interquartile range, 8-10). Intraclass correlation coefficients between e-ASPECTS and raters were $0.72,0.74$, and 0.76 (all, $P<.001$ ). e-ASPECTS (Spearman rank correlation coefficient $=-0.15, P=.027$ ) correlated with mRS at 3 months. Patients with unfavorable outcome had lower e-ASPECTS (median, 8; interquartile range, 7-10 versus median, 9; interquartile range, $8-10 ; P=.014)$. Sixteen patients (7.4\%) had a poor scan, which was associated with unfavorable outcome (OR, 13.6; $95 \% \mathrm{Cl}, 1.8-104)$. Independent predictors of unfavorable outcome were e-ASPECTS (OR, 0.79; 95\% Cl, 0.63-0.99), blood sugar (OR, 1.01; 95\% Cl, 1.004-1.02), atrial fibrillation (OR, 2.64; 95\% Cl, 1.22-5.69), premorbid mRS (OR, 1.77; 95\% Cl, 1.21-2.58), NIHSS (OR, 1.11; 95\% Cl, 1.04-1.19), general anesthesia (OR, 0.24; 95\% Cl, 0.07-0.84), failed recanalization (OR, 8.47; 95\% Cl, 3.5-20.2), and symptomatic intracerebral hemorrhage (OR, 25.8; 95\% Cl, 2.5-268).

CONCLUSIONS: The e-ASPECTS correlated with mRS at 3 months and was predictive of unfavorable outcome after mechanical thrombectomy, but further studies in patients with poor scan are needed.

ABBREVIATIONS: AIS = acute ischemic stroke; $M T$ = mechanical thrombectomy; sICH = symptomatic intracranial hemorrhage

M echanical thrombectomy (MT) in acute ischemic stroke (AIS) due to a large intracranial vessel occlusion in the anterior circulation has been proven to be an effective therapy. ${ }^{1-5}$ With the success of the recent trials, an increasing number of patients with AIS will be evaluated for eligibility for this therapy. However, because there are no strict ex-/inclusion guidelines, ${ }^{6}$ patient selection for MT remains a challenging process. In the

Received January 12, 2017; accepted after revision March 22.

From the Departments of Neuroradiology (J.P., C.H., M.M., M.B.) and Neurology (S. Shieber, S. Schönenberger, J.B., P.A.R., S.N.), University of Heidelberg, Heidelberg, Germany.

Please address correspondence to Simon Nagel, MD, Universität Heidelberg, Abteilung für Neurologie, Im Neuenheimer Feld 400, 69120 Heidelberg, Germany; e-mail: simon.nagel@med.uni-heidelberg.de

- Indicates open access to non-subscribers at www.ajnr.org

三 Indicates article with supplemental on-line table.

http://dx.doi.org/10.3174/ajnr.A5236 end, this evaluation process should not withhold therapy from patients who might benefit from MT but should preclude patients who will not benefit. During the evaluation process, multiple factors need to be cautiously reviewed. Noncontrast CT is still the most available imaging tool worldwide. Early signs of infarction on NCCT scans are a potent predictor of clinical outcome in patients with AIS. ${ }^{7}$ In 2000, Barber et al $^{8}$ introduced the Alberta Stroke Program Early CT Score. ASPECTS divides the middle cerebral artery territory into 10 predefined anatomic areas and grades the presence of early infarct signs by parenchymal hypodensity on NCCT. This increasingly used score has already proved a reliable predictor of clinical outcome in patients with AIS treated with intravenous $\mathrm{rtPA}$ and/or MT. ${ }^{8-10}$

Unfortunately, the determination of early signs of ischemia and their translation into the ASPECTS have a considerable interrater variability, which is, among other factors, influenced by rater experience. ${ }^{11-13}$ However, during the evaluation process for per- 
forming MT or before transfer of a patient to a comprehensive stroke center performing MT, scoring variability could negatively influence the decision-making process and lead to false patient in-/exclusion. The e-ASPECTS software (Brainomix, Oxford, UK; www.brainomix.com) is a standardized, fully automated, CE mark-approved ASPECTS scoring tool for NCCT, which has previously demonstrated scoring on an expert level. ${ }^{14,15}$ We analyzed whether e-ASPECT scores correlate with outcome after mechanical thrombectomy and whether low scores are predictors of unfavorable outcome. In particular, we were interested in whether patients with low e-ASPECTS ( $0-5$, poor scans) were very likely to have a bad outcome.

\section{MATERIALS AND METHODS}

From a prospectively collected stroke data base, we identified 430 patients with AIS in the anterior circulation who underwent MT between May 2010 and May 2015. All patients were treated with endovascular revascularization therapy and, in case of eligibility, with intravenous thrombolysis after an interdisciplinary decision by the neurologist and neurointerventionalist on call. Historically, during MT, patients were treated under general anesthesia; and since 2009, we have been following a strict standardized operating procedure. In September 2013, an additional standardized operating procedure for conscious sedation was introduced, and selected patients were treated following this procedure with MT. Since April 2014, eligible patients were included in our singlecenter randomized sedation trial, Sedation vs. Intubation for Endovascular Stroke TreAtment (SIESTA). ${ }^{16}$ After the procedure, all patients were treated according to in-house standardized operating procedures in our neurointensive care unit and/or our stroke unit. In our data base, clinical data at baseline and during the hospital stay as well as radiologic and laboratory information are prospectively entered and used for analysis when appropriate.

Recanalization was assessed with the Thrombolysis in Cerebral Infarction Score by a blinded investigator (J.P.). TICI 0-2a was scored as failed, and TICI 2b-3 was scored as successful recanalization. The outcome was measured with the modified Rankin Scale after 3 months and was obtained through rehabilitation reports, outpatient assessments, or a standardized interview by an unblinded investigator. Unfavorable outcome was defined as a modified Rankin Scale score between 4 and 6; favorable outcome, as mRS $0-3$; and good outcome, as mRS 0-2. Symptomatic intracranial hemorrhage ( $\mathrm{sICH}$ ) was defined according to the European Cooperative Acute Stroke Study II (blood at any site in the brain and clinical deterioration with an increase in the NIHSS score of at least 4 points compared with the lowest value within the first 7 days or any intracranial hemorrhage leading to death).

NCCT scans of the included patients were obtained from the same multisection CT scanners. Between May 2010 and January 2014, images were acquired from a Somatom Volume Zoom (Siemens, Erlangen, Germany, $n=150$ ). Incremental sections had a thickness of $6 \mathrm{~mm}$ with an in-plane resolution of $512 \times 512 \mathrm{~mm}$. Between February 2014 and May 2015, NCCTs were acquired from a Somatom Definition AS (Siemens, $n=70$ ) with a section thickness of $4 \mathrm{~mm}$ and an in-plane resolution of $512 \times 512 \mathrm{~mm}$. NCCT scans were retrospectively scored by e-ASPECTS (Version 5.0), as well as by 3 stroke experts, and software and experts were blinded to any patient data and clinical information except for the side of the unilateral ischemic stroke. The experts had 6-12 years of extensive experience in neurovascular imaging interpretation (J.P. and C.H., board-certified neuroradiologists; S.N., boardcertified neurologist). e-ASPECTS is based on a combination of advanced image-processing and machine-learning algorithms. Several image-enhancement filters are applied to the input DICOM CT image to deal with noise, differences between scanners, and image artifacts. A 3D registration module corrects for any tilt, rotation, and other transformations. The ASPECTS regions are then segmented; this segmentation provides a standardized reference for the cortical regions. The scoring module operates on the standardized 3D images, classifying signs of ischemic damage and assigning them to ASPECTS regions. It applies statistical learning methods to image features to determine whether a region is likely to be damaged. ${ }^{14,15}$ An e-ASPECTS score between 0 and 5 was considered a poor scan.

\section{Statistical Analysis}

Our primary end point was defined as unfavorable outcome as opposed to good outcome because we wanted to test the hypothesis that patients with low e-ASPECTS are indeed under a higher risk of futile treatment. Nominal data are presented as frequencies; ordinal data, as median with interquartile range; and metric data, as mean \pm SD. To show univariate differences in outcome groups, we used the Fisher exact test, the $\chi^{2}$ test, the Mann-Whitney $U$, or unpaired $t$ test when appropriate according to data category. For multivariate analysis to show predictors of unfavorable and good outcome, we used a stepwise forward binary logistic regression model with correction for all significant variables $(P \leq$ $.05)$ in univariate analyses. Odds ratios are presented as values with 95\% confident intervals. For the agreement between eASPECTS with each expert we used the intraclass correlation coefficient. For correlation of the e-ASPECTS and the mRS at 3 months, we used the Spearman rank correlation coefficient.

\section{Ethics Approval}

Our local ethics committee approved the prospective data base and retrospective analysis herein. Due to its monocentric and retrospective character, the requirement for subsequent written patient informed consent was waived.

\section{RESULTS}

Two hundred twenty patients of 430 met the inclusion criteria for this analysis (ie, in-house NCCT with no or minor motion artifacts before MT; Fig 1). Patients' baseline characteristics are shown in the On-line Table. This shows that our cohort is very typical for patients with acute large intracranial vessel occlusion. The median NIHSS at baseline was 18 (interquartile range, 1521 ), but in contrast to previous randomized controlled trials, we also included around $9 \%$ of patients with a premorbid $\mathrm{mRS}$ of $>2$ (ie, 14 patients with an mRS of 3 and 6 with an mRS of 4). Most patients had either an $\mathrm{M} 1$ occlusion or a carotid-T occlusion. One hundred forty-seven (67\%) patients were treated with rtPA-based bridging protocols. After prior exclusion of $43(10 \%)$ patients due to severe motion artifacts rendering ASPECTS scoring for the 


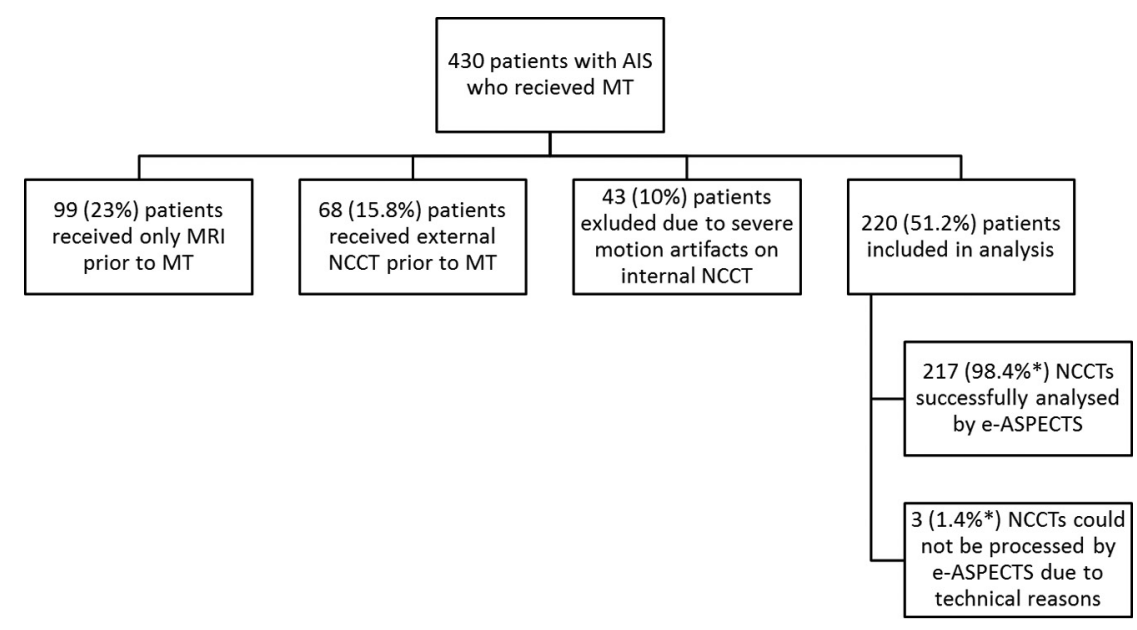

FIG 1. Exclusion criteria for patients with AIS who received MT entering this analysis. Severe motion artifacts were defined as artifacts that impair differentiation of anatomic structures (eg, basal ganglia, subarachnoid space) and/or clear identification of intracranial hemorrhage by the human rater. The asterisk indicates percentage regarding included patients.

human rater impossible, an additional 3 (1.4\%) scans could not be processed by e-ASPECTS due to technical reasons (upload or processing of the DICOM data). The median e-ASPECTS was 9 (interquartile range, $8-10$ ) The distribution of e-ASPECTS and human raters' scores are shown in Fig 2. Only 16 patients (7.4\%) had a poor scan, which was strongly associated with unfavorable outcome (OR, 13.6; 95\% CI, 1.8-104; $P=.012)$. Overall, after 3 months, the primary end point of unfavorable outcome was met by 123 (55.9\%) patients, and $61(27.7 \%)$ patients reached the secondary end point of good outcome.

The intraclass correlation coefficients between e-ASPECTS and each rater were $0.72,0.74$, and 0.76 , respectively (all, $P<$ $.001)$. The agreement within the human raters was very robust as well (intraclass correlation coefficients, 0.7, 0.77, and 0.75; all, $P<$ .001). Although the overall agreement of raters and e-ASPECTS was good, in some cases, considerably different scoring results occurred and an example with a low e-ASPECTS (4) is presented in Fig 3. Only e-ASPECTS (Spearman rank correlation coefficient $=-0.15, P=.027)$ and the expert's scoring with the highest agreement with e-ASPECTS (Spearman rank correlation coefficient $=-0.19, P=.004$ ) correlated inversely but significantly with $\mathrm{mRS}$ at 3 months, indicating that a lower ASPECTS is associated with a higher mRS after 3 months. ASPECTS scoring of the other 2 readers differed from the e-ASPECTS scores to the extent that a significant statistical correlation between their ASPECTS scores and clinical outcome could not be detected. Spearman rank correlation coefficient for e-ASPECTS and mRS in our analysis differed from the Spearman rank correlation coefficient in the study of Barber et al $(-0.69)^{8}$ because Barber et al used only 3 categories of the mRS for correlation and their cohort consisted of consecutive patients with suspected stroke in the anterior circulation, whereas our patients all had proved large-vessel occlusion.

One hundred ninety-eight (90\%) patients were treated with modern stent retrievers (mostly Solitaire; Covidien, Irvine, California), and 195 (88.6\%) patients underwent thrombectomy under general anesthesia. In the univariate analysis for the primary end point, among other significant differences, patients with unfavorable outcome had lower e-ASPECTS (median, 8; interquar- tile range, 7-10 versus median, 9; interquartile range, $8-10 ; P=.014$; On-line Table). Bridging therapy or treatment and procedural times, however, were not significantly different in patients with unfavorable outcome versus patients with favorable outcome (mRS, 0-3). For multivariate analysis, we included the variables age, premorbid mRS, atrial fibrillation, previous stroke, baseline blood sugar, and hemoglobin A1c, as well as NIHSS, hyperdense vessel sign, e-ASPECTS, general anesthesia, recanalization, and sICH (all $P<.05$ in univariate analysis). Independent predictors of unfavorable outcome were eASPECTS (OR, 0.79; 95\% CI, 0.63-0.99; $P=.049$ ) but also blood sugar (OR, 1.01; 95\% CI, 1.004-1.02; $P=.005)$, atrial fibrillation (OR, 2.64; 95\% CI, $1.22-5.69 ; P=.013$ ), premorbid mRS (OR, 1.77; 95\% CI, $1.21-$ $2.58 ; P=.003)$, NIHSS (OR, $1.11 ; 95 \% \mathrm{CI}, 1.04-1.19 ; P=.003)$, general anesthesia (OR, $0.24 ; 95 \% \mathrm{CI}, 0.07-0.84 ; P=.007)$, failed recanalization (OR, 8.47; 95\% CI, 3.5-20.2; $P<.001$ ), and $\mathrm{sICH}$ (OR, 25.8; 95\% CI, 2.5-268; $P=.007$ ).

In the analysis of the secondary end point, factors associated with good outcome were mean age ( $66 \pm 12$ versus $74 \pm 11$ years; $P<.001$ ), premorbid $\mathrm{mRS}$ (median, 0 ; interquartile range, $0-1$ versus median, 1 ; interquartile range, $0-2 ; P=.002$ ), atrial fibrillation (34.4\% versus 52.2\%; $P=.023)$, blood sugar $(118 \pm 33$ versus $139 \pm 57 \mathrm{mg} / \mathrm{dL} ; P=.015)$, creatinine $(0.89 \pm 0.25$ versus $1.1 \pm 0.78 \mathrm{mg} / \mathrm{dL} ; P=.03)$, general anesthesia $(80.3 \%$ versus $91.8 \% ; P=.03$ ), NIHSS (median, 16; interquartile range, 11-19 versus median, 19 ; interquartile range, $15-22 ; P<.001)$, successful recanalization $(88.5 \%$ versus $58.9 \%, P<.001)$ and $\mathrm{sICH}(0 \%$ versus $8.8 \%, P=.012$ ). The e-ASPECTS, however, was not associated with good outcome (median, 9; interquartile range, 8-10 versus median, 9; interquartile range, $7-10 ; P=.249$ ). After multivariate analysis (sICH was not included in the model because no patient with good outcome had sICH), only age (OR, 0.96; 95\% CI, 0.93-0.98), blood sugar levels (OR, 0.99; 95\% CI, 0.99-1.0), NIHSS (OR, 0.91; 95\% CI, 0.86-0.97), and successful recanalization (OR, 5.45; 95\% CI, 2.18-13.61) remained independent predictors of good outcome.

\section{DISCUSSION}

Our study confirmed that the e-ASPECTS software performs in a manner similar to that of stroke experts in detecting early ischemic damage in the MCA territory. Furthermore, we could demonstrate for the first time that fully automatically derived eASPECT scores correlated inversely with patient outcome 3 months after MT (Spearman rank correlation coefficient $=-0.15, P=$ .027). Patients with poor scans (ASPECTS $0-5$ ) at baseline had a significantly high probability of unfavorable outcome (OR, 13.6; 95\% CI, 1.8-104). On the basis of the multivariate regression analysis, a 1-point decrease in the e-ASPECTS increases the risk for an unfavorable clinical outcome by $20 \%$. Most important, indicating the robustness of our results, we could also confirm 

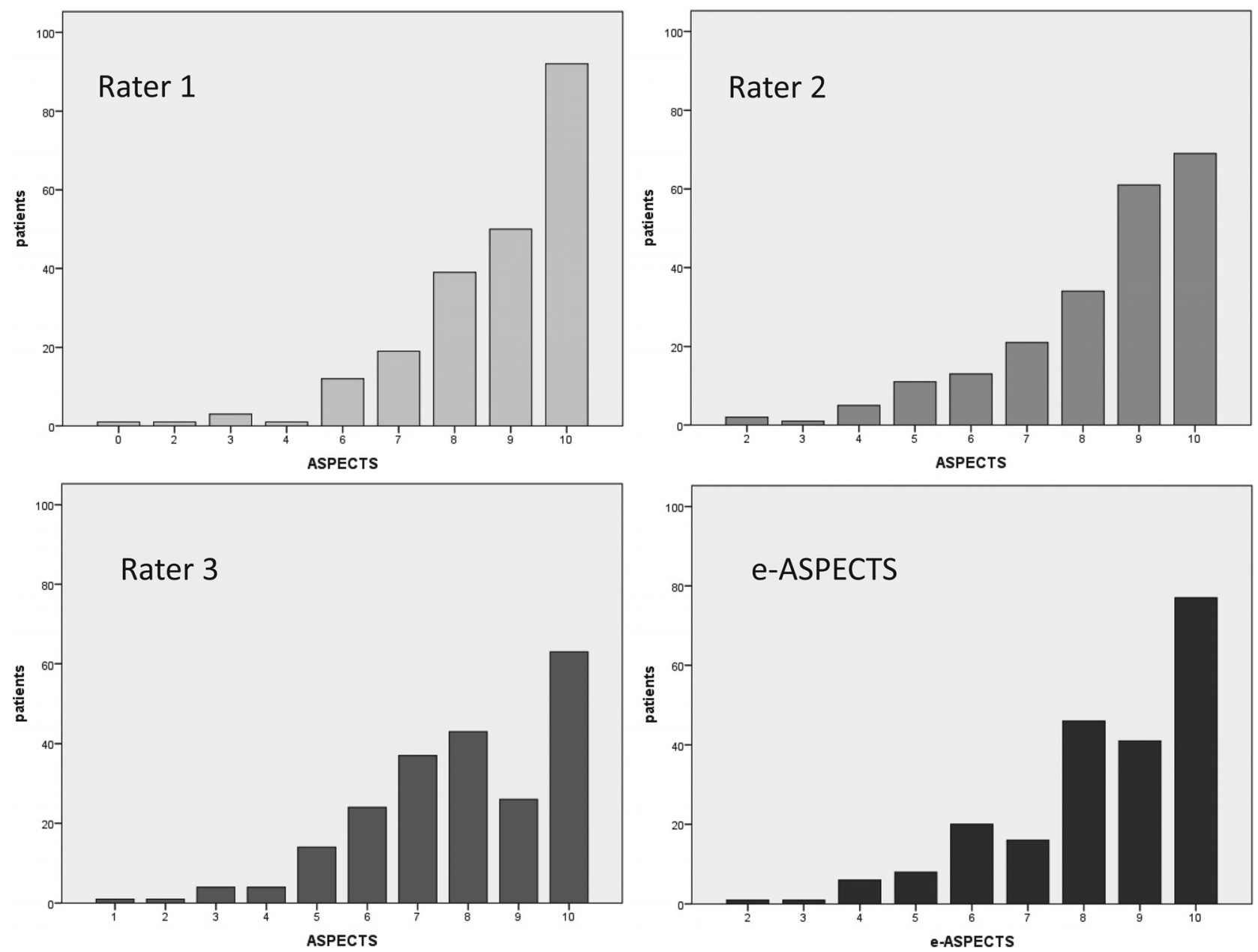

FIG 2. Distribution of ASPECTSs according to the 3 human raters and e-ASPECTS.

other known predictors of outcome after acute ischemic stroke, such as recanalization, sICH, atrial fibrillation, NIHSS, premorbid functionality, and blood sugar levels.

The ASPECTS is a validated, reliable, and reproducible grading system to assess early ischemic changes on noncontrast CT studies in patients with acute ischemic stroke of the anterior circulation. ${ }^{8}$ The Highly Effective Reperfusion evaluated in Multiple Endovascular Stroke trials (HERMES) collaboration has recently shown a clear association between favorable clinical outcome after MT and ASPECTS of $\geq 6 .{ }^{17}$ Additionally, a subgroup analysis of the Multicenter Randomized Clinical trial of Endovascular treatment for Acute ischemic stroke in the Netherlands (MR CLEAN) also demonstrated that patients with moderate early ischemic changes (ASPECTS 5-7) benefit from mechanical thrombectomy. ${ }^{18}$ On the other hand, there are other studies that could not show a correlation between ASPECTS 5-7 and clinical outcome after 3 months, ${ }^{19}$ and the HERMES data revealed that there is no clear benefit for patients with an ASPECTS of 0-5. Another study found that patients with ASPECTS 0-4 before mechanical thrombectomy had higher rates of serious adverse events and hemicraniectomy. Furthermore, death within 1 week and 30 days after treatment was more frequent. ${ }^{18}$ Therefore, the effect of endovascular treatment for patients with a poor scan (ASPECTS $0-5$ ) is unclear, with perhaps only a slim margin for benefit. Ad-

ditionally, multivariate regression analysis to show the independent prognostic effects of the ASPECTS has not been performed in these previous trials.

Because the probability of achieving functional independence declines and the risk of severe disability and death rises with lower ASPECTS, this scoring system appears important for patient evaluation for endovascular stroke treatment. Our data indicate that e-ASPECTS can provide important technical support in estimating a patient's prognosis. Furthermore, in the present analysis, 2 of 3 expert raters' scores did not correlate with the clinical outcome, whereas e-ASPECT scores did. This nicely illustrates 2 features: First, as already shown by others, the ASPECTS has considerable variability even among experts. This may be even more pronounced in the acute treatment decision for understandable reasons, such as emotional issues and stress. Second, an automated scoring tool can be advantageous here due to its "objectivity." Hence, e-ASPECTS could contribute to decision-making for patient selection in the community hospital or telemedicine services and/or patient transfer into a comprehensive stroke center for MT. Other important imaging variables derived from CT angiography and CT perfusion have also been shown to carry prognostic information that can inform treatment decision-making, ${ }^{20}$ but NCCT is still the most commonly used imaging tool in acute stroke. 


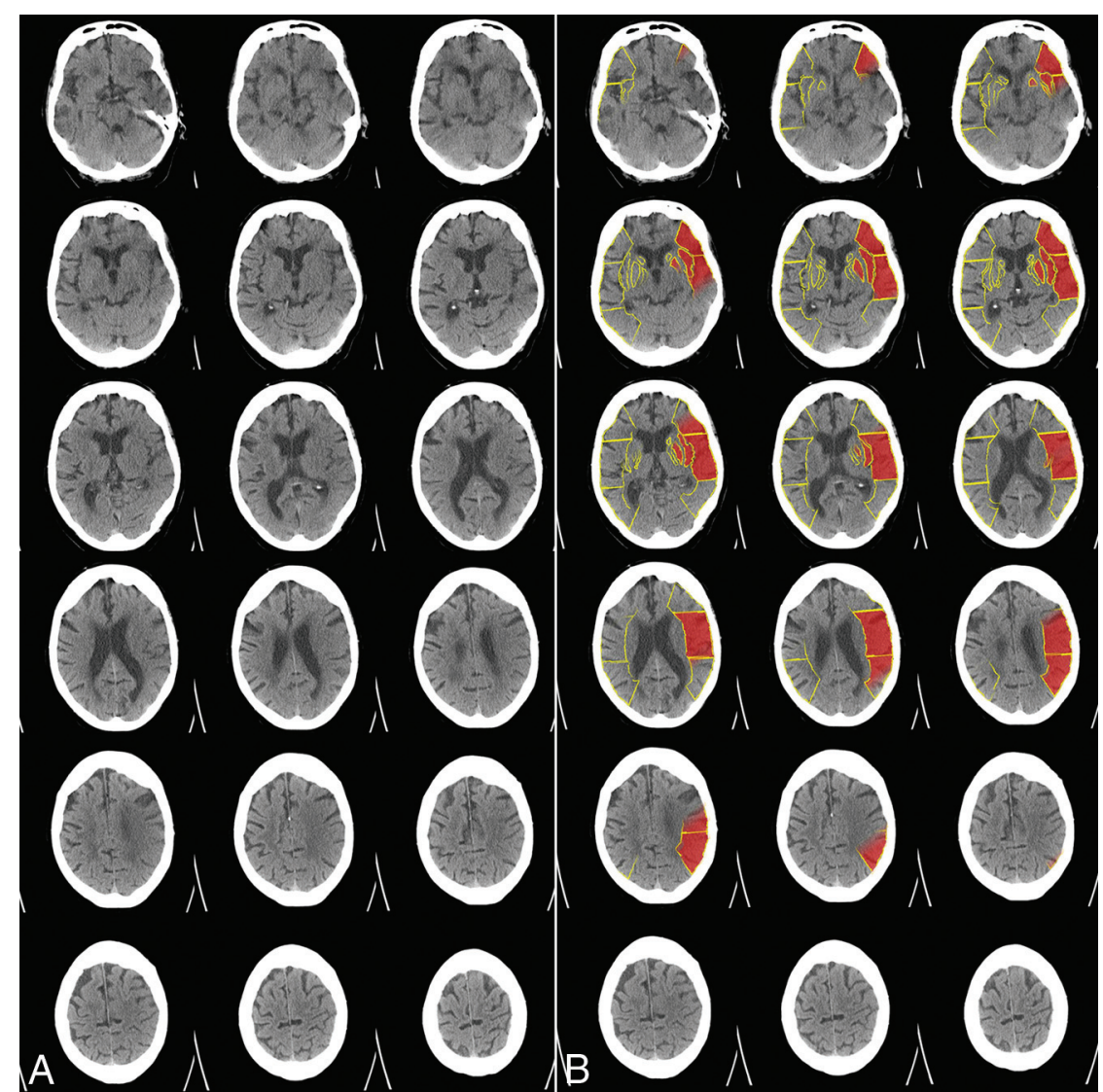

FIG 3. Example of a patient with extensive early signs of ischemic damage on the baseline NCCT (A). The processed e-ASPECTS results is a score of 4 . The human raters scored an ASPECTS of 6, 8, and 9 , respectively, highlighting considerable disagreement in this particular case (B). An M1 occlusion was present, onset to treatment time was 143 minutes, TICI $2 \mathrm{a}$ was achieved, and no sICH occurred. Unfortunately, this patient had died within 3 months.

Our analysis focused on the prognostic value of ASPECTS, specifically e-ASPECTS for unfavorable outcome, as opposed to good outcome. According to current guidelines considering endovascular stroke therapy, a favorable ASPECTS is not an unconditional prerequisite, especially within 6 hours from onset (American Heart Association guidelines/European Stroke Organisation-European Foundation of Minimally Invasive Neurological Therapy guidelines). ${ }^{21,22}$ The question does not seem to be whom to treat but for whom treatment could be disadvantageous due to missing success or the increased likelihood of complications because patients with ASPECTS $\leq 5$ have a lower chance of independent living or even survival. The eASPECTS was not a predictor of good outcome in this analysis, which indicates that patients with low scores still have a considerable probability of achieving clinical improvement through therapy; therefore, this analysis reinforces the need for a prospective controlled trial to investigate the potential benefit of thrombectomy in patients with a low initial ASPECTS.

Our study has several limitations: 1) Because we treated patients with broad inclusion criteria (ie, higher premorbid mRS and so forth), one cannot expect outcome results similar to those in the recent seminal MT trials, but rather similar to real-life data. 2) This analysis was based on a prospective single-center cohort data base, and data were analyzed retrospectively; therefore, caution should be exercised when generalizing the results. Our data regarding the mode of sedation need to be interpreted with caution. Most $(88.6 \%)$ patients were treated under general anesthesia; and only a minority, with conscious sedation. We only introduced this mode of sedation in September 2013, and until April 2014, a selection bias in our cohort was present. The results of the SIESTA trial, however, indicated that in our center, general anesthesia is not associated with impaired outcome. $^{16}$ 3) The performance of e-ASPECTs might have been compromised due to the available section thicknesses of 4 and $6 \mathrm{~mm}$; the optimal thickness for analysis is $1 \mathrm{~mm}$. Moreover, there was no ground truth (ie, diffusionweighted imaging or CT perfusion) defined for ASPECTS scoring for this analysis. However, the e-ASPECTS software has previously shown similar and noninferior performance to that of stroke experts, ${ }^{14,15}$ and most centers do not have sophisticated imaging tools and rely on NCCT, which makes this an important analysis. 4) Additionally, the number of patients with a poor scan of $\leq 5$ ASPECTS points was low, and our results need to be confirmed by analysis of large registries of patients undergoing MT, at best by randomized controlled trials including those patients in whom considerable uncertainty still exists with respect to the efficacy and safety of MT (ie, patients with extensive ischemic brain damage at baseline). The e-ASPECTS software could be a suitable tool for patient stratification in such trials.

The strengths of this study were that our cohort was very typical for patients undergoing MT and that we included numerous clinical, laboratory, and radiologic data in our analysis. The multivariate model confirmed many previously described prognostic factors, while the predictive value of e-ASPECTS prevailed, thereby indicating the robustness of our results.

\section{CONCLUSIONS}

The e-ASPECTS correlated with outcome overall and was predictive of unfavorable outcome after mechanical thrombectomy. e-ASPECTS might facilitate treatment decision-making, especially in centers without modern imaging tools for tissue perfusion and collateral status. It is easy to use, has a robust and reliable performance, and removes intra- and interrater variability. Because there are few patients with poor initial CT scans who improve after treatment, further prospective studies in those patients are needed and individual decision-making remains necessary.

Disclosures: Johannes Pfaff_-UNRELATED: Payment for Lectures Including Service on Speakers Bureaus: Siemens; Travel/Accommodations/Meeting Expenses Unre- 
lated to Activities Listed: Stryker. Christian Herweh-UNRELATED: Consultancy: Brainomix, Comments: temporary consultancy related to a project different from the one from which the current article originated; Payment for Lectures Including Service on Speakers Bureaus: Bristol-Myers Squibb, Brainomix: once in 2016; Travel/ Accommodations/Meeting Expenses Unrelated to Activities Listed: Stryker, Brainomix: travel expenses for attending a national meeting in 2016. Julian BöselUNRELATED: Grants/Grants Pending: Patient-Centered Outcomes Research Institute, Brainomix Ltd: award pending for SETPOINT2 Trial; Travel/Accommodations/ Meeting Expenses Unrelated to Activities Listed: Zoll, Bard Medical, Sedana Medical, Seiratherm, Boehringer Ingelheim, Brainomix Ltd: speaker's honoraria and travel support. Peter A. Ringleb_UNRELATED: Consultancy: advisory board member for Boehringer Ingelheim, Covidien; Payment for Lectures Including Service on Speakers Bureaus: lecture fee from Boehringer Ingelheim, Bayer, Pfizer, Daichii Sanyo. Markus Möhlenbruch-UNRELATED: Payment for Lectures Including Service on Speakers Bureaus: Codman, MicroVention, phenox, Stryker. Martin Bendszus-UNRELATED: Board Membership: Data and Safety Monitoring Board for Vascular Dynamics; Consultancy: Codman, Roche, Guerbet, Boehringer Ingelheim; Grants/Grants Pending: Deutsche Forschungsgemeinschaft, Hopp Foundation, Novartis, Siemens, Guerbet, Stryker, Covidien*; Payment for Lectures Including Service on Speakers Bureaus: Novartis, Roche, Guerbet, Teva Pharmaceutical Industries, Bayer, Codman. Simon Nagel-UNRELATED: Consultancy: Brainomix; S.N. has had an ongoing consultancy appointment with Brainomix since 2015 independent from the current study; Grants/Grants Pending: Brainomix; The University Hospital Heidelberg received grants from Brainomix for prior studies*; Payment for Lectures Including Service on Speakers Bureaus: Bayer, Medtronic, Pfizer; Travel/Accommodations/Meeting Expenses Unrelated to Activities Listed: Boehringer Ingelheim, Brainomix. *Money paid to the institution.

\section{REFERENCES}

1. Berkhemer OA, Fransen PSS, Beumer D, et al. A randomized trial of intraarterial treatment for acute ischemic stroke. $N$ Engl J Med 2015; 372:11-20 CrossRef Medline

2. Campbell BC, Mitchell PJ, Kleinig TJ, et al; EXTEND-IA Investigators. Endovascular therapy for ischemic stroke with perfusion-imaging selection. $N$ Engl J Med 2015;372:1009-18 CrossRef Medline

3. Goyal M, Fargen KM, Turk AS, et al. 2C or not 2C: defining an improved revascularization grading scale and the need for standardization of angiography outcomes in stroke trials. J Neurointerv Surg 2014;6:83-86 CrossRef Medline

4. Jovin TG, Chamorro A, Cobo E, et al; REVASCAT Trial Investigators. Thrombectomy within $\mathbf{8}$ hours after symptom onset in ischemic stroke. N Engl J Med 2015;372:2296-306 CrossRef Medline

5. Saver JL, Goyal M, Bonafe A, et al; SWIFT PRIME Investigators. Stent-retriever thrombectomy after intravenous t-PA vs. t-PA alone in stroke. $N$ Engl J Med 2015;372:2285-95 CrossRef Medline

6. A collaboration of the European Stroke Organisation-Karolinska Stroke Update, European Society for Minimally Invasive Neurological Therapy, and European Society of Neuroradiology. Consensus statement on mechanical thrombectomy in acute ischemic stroke. In: Proceedings of the European Stroke Organisation-Karolinska Stroke Update, Stockholm, Sweden. November 16-18, 2014

7. Wardlaw JM, Mielke O. Early signs of brain infarction at CT: observer reliability and outcome after thrombolytic treatmentsystematic review. Radiology 2005;235:444-53 CrossRef Medline

8. Barber PA, Demchuk AM, Zhang J, et al. Validity and reliability of a quantitative computed tomography score in predicting outcome of hyperacute stroke before thrombolytic therapy: ASPECTS Study Group-Alberta Stroke Programme Early CT Score. Lancet 2000; 355:1670-74 CrossRef Medline

9. Menon BK, Puetz V, Kochar P, et al. ASPECTS and other neuroimaging scores in the triage and prediction of outcome in acute stroke patients. Neuroimaging Clin N Am 2011;21:407-23, xii CrossRef Medline
10. Liebeskind DS, Jahan R, Nogueira RG, et al; SWIFT Investigators. Serial Alberta Stroke Program Early CT score from baseline to 24 hours in Solitaire Flow Restoration with the Intention for Thrombectomy study: a novel surrogate end point for revascularization in acute stroke. Stroke 2014;45:723-27 CrossRef Medline

11. Demaerschalk BM, Silver B, Wong E, et al. ASPECT scoring to estimate $>1 / 3$ middle cerebral artery territory infarction. Can J Neurol Sci 2006;33:200-04 CrossRef Medline

12. Mak HK, Yau KK, Khong PL, et al; Alberta Stroke Programme Early CT Score. Hypodensity of $>1 / 3$ middle cerebral artery territory versus Alberta Stroke Programme Early CT Score (ASPECTS): comparison of two methods of quantitative evaluation of early CT changes in hyperacute ischemic stroke in the community setting. Stroke 2003;34:1194-96 CrossRef Medline

13. Finlayson $\mathrm{O}$, John $\mathrm{V}$, Yeung $\mathrm{R}$, et al. Interobserver agreement of ASPECT score distribution for noncontrast CT, CT angiography, and CT perfusion in acute stroke. Stroke 2013;44:234-36 CrossRef Medline

14. Herweh C, Ringleb PA, Rauch G, et al. Performance of e-ASPECTS software in comparison to that of stroke physicians on assessing CT scans of acute ischemic stroke patients. Int J Stroke 2016;11:438-45 CrossRef Medline

15. Nagel S, Sinha D, Day D, et al. e-ASPECTS software is non-inferior to neuroradiologists in applying the ASPECTS score to CT scans of acute ischemic stroke patients. In: Proceedings of the 2nd European Stroke Organisation Conference, Barcelona, Spain. May 10-12, 2016

16. Schonenberger S, Uhlmann L, Hacke W, et al. Effect of conscious sedation vs general anesthesia on early neurological improvement among patients with ischemic stroke undergoing endovascular thrombectomy: a randomized clinical trial. JAMA 2016;316: 1986-96 CrossRef Medline

17. Goyal M, Menon BK, van Zwam WH, et al; HERMES collaborators Endovascular thrombectomy after large-vessel ischaemic stroke: a meta-analysis of individual patient data from five randomised trials. Lancet 2016;387:1723-31 CrossRef Medline

18. Yoo AJ, Berkhemer OA, Fransen PS, et al; MR CLEAN investigators. Effect of baseline Alberta Stroke Program Early CT Score on safety and efficacy of intra-arterial treatment: a subgroup analysis of a randomised phase 3 trial (MR CLEAN). Lancet Neurol 2016;15: 685-94 CrossRef Medline

19. Costalat V, Lobotesis K, Machi P, et al. Prognostic factors related to clinical outcome following thrombectomy in ischemic stroke (RECOST study): 50 patients prospective study. Eur J Radiol 2012; 81:4075-82 CrossRef Medline

20. Turk AS, Magarick JA, Frei D, et al. CT perfusion-guided patient selection for endovascular recanalization in acute ischemic stroke: a multicenter study. J Neurointerv Surg 2013;5:523-27 CrossRef Medline

21. Powers WJ, Derdeyn CP, Biller J, et al; American Heart Association Stroke Council. 2015 American Heart Association/American Stroke Association Focused Update of the 2013 Guidelines for the Early Management of Patients with Acute Ischemic Stroke Regarding Endovascular Treatment: A Guideline for Healthcare Professionals from the American Heart Association/American Stroke Association. Stroke 2015;46:3020-35 CrossRef Medline

22. Wahlgren N, Moreira T, Michel P, et al; ESO-KSU, ESO, ESMINT, ESNR and EAN. Mechanical thrombectomy in acute ischemic stroke: consensus statement by ESO-Karolinska Stroke Update 2014/2015, supported by ESO, ESMINT, ESNR and EAN. Int J Stroke 2016;11:134-47 CrossRef Medline 